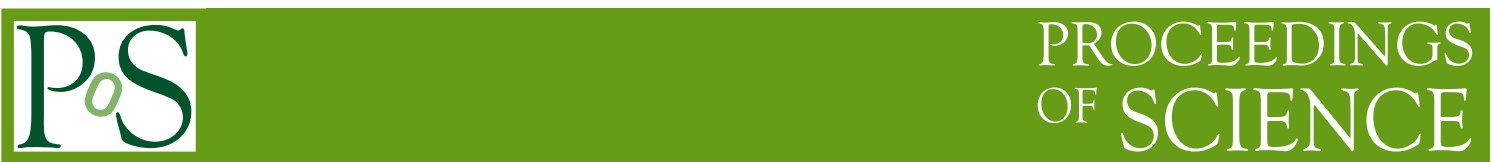

\title{
Performance of the LHCb detector in Run 2
}

\author{
Martina Pili ${ }^{a, 1, *}$ \\ ${ }^{a}$ University of Oxford, \\ Particle Physics Sub-department, Keble Road, Oxford, OX1 3RH, United Kingdom \\ E-mail: martina.pili@cern.ch
}

The LHCb experiment is a flavour physics detector, designed to study decays of $b$ and $c$ hadrons for measurements of CP violation and rare decays. Its performance is based on precision tracking and particle-identification systems. In order to accomplish its wide program of physics measurements, the LHCb collaboration has developed in the past years a set of algorithms for reconstruction of the trajectories of charged particles, as well as identification of charged and neutral particles. Several data-driven approaches have been developed to provide a precise calibration of the tracking and particle-identification efficiencies, which are crucial ingredients of many physics analyses. A number of novel strategies have been developed during Run 2 of the LHC to improve the precision of this calibration. This talk presents an overview of the LHCb performance in Run 2 of the LHC, with emphasis on recent improvements.

40th International Conference on High Energy physics - ICHEP2020

July 28 - August 6, 2020

Prague, Czech Republic (virtual meeting)

${ }^{1}$ On behalf of the LHCb Collaboration.

* Speaker 


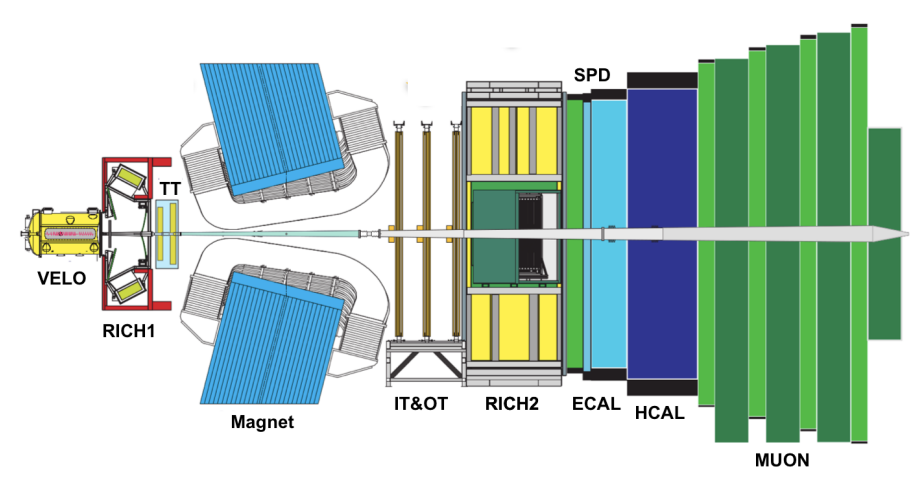

Figure 1: Schematic view of the LHCb subdetectors [1].

\section{Introduction}

The LHCb detector is a single-arm forward spectrometer covering the pseudorapidity range $2<\eta<5$ [1]. The detector configuration in Run 2 includes a high-precision tracking system consisting of a silicon-strip vertex detector (VELO) for precise determination of the position of primary and secondary interaction vertices, a large area silicon strip detector (TT) located upstream of a dipole magnet with a bending power of about $4 \mathrm{~T} \cdot \mathrm{m}$, and three stations of silicon-strip detectors (IT) and straw drift tubes (OT) placed downstream of the magnet denoted as T1-T3 stations. The particle identification system is composed of two Ring Imaging Cherenkov detectors (RICH1, RICH2), electromagnetic (ECAL) and hadronic (HCAL) calorimeters, and a system of muon chambers (MUON). A scheme of the subdetectors described above is shown in Fig. 1.

Precise detector alignment and calibration is crucial for the LHCb physics programme, to avoid degradation in the mass resolution and maximise the performance of the particle identification system (PID). The excellent performance achieved in Run 2 are made possible by a novel computing strategy introduced in the year 2015 [2]. LHCb is the first experiment to perform a real time detector alignment and calibration at trigger level, with full event reconstruction taking place at the second stage of the software trigger (denoted by HLT2). The quality of the online reconstruction matches that of the offline reconstruction. This allows for some analyses to run directly from the output of the trigger, by using the so-called Turbo stream; this strategy also allows the selection of high purity and high statistics calibration samples, unbiased by any other triggers, useful to study tracking and PID performance. A dedicated set of trigger lines grouped in the TurboCalib stream has been developed for this purpose.

\section{Tracking performance}

In Run 2, the alignment procedure for the LHCb detector was performed for each fill in real time [3]. The alignment is based on the $\chi^{2}$ minimisation of the residuals evaluated with the Kalman 
filter fit taking into account the multiple scattering and the energy loss corrections [4]. In addition, this method offers the possibility to apply mass and vertex constraints. The sample used for the alignment was collected in few minutes at the beginning of each fill. It was a minimum bias sample applying a vertex constraint for the VELO and a $D^{0}$ two-body decay sample with a mass constraint applied to the $D^{0}$ candidates for the tracker. During 2017, an alignment was performed using the full $Z$ sample collected during 2016 with a mass constraint applied to the $Z$ candidates. The results obtained with higher momentum tracks result in a more precise alignment and allow to align some elements not aligned in the real time procedure. This alignment was used as starting point of the real-time alignment procedure since end of June 2018. Figure 2 compares the reconstructed dimuon invariant mass distribution in a sample of $Z \rightarrow \mu^{+} \mu^{-}$data, before and after the application of the $Z$ alignment. A $35 \%$ improvement in the mass resolution is observed.

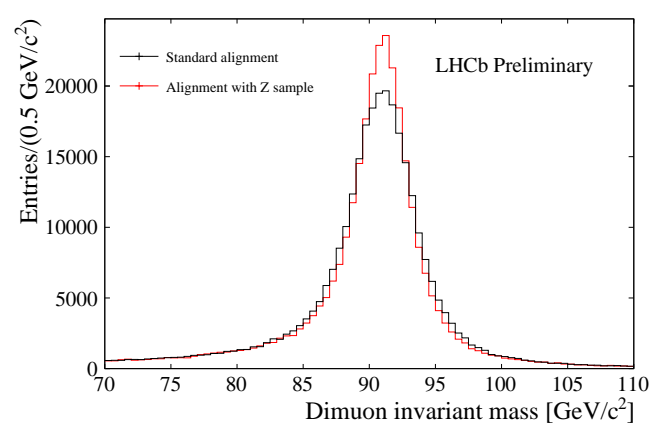

Figure 2: The invariant mass distribution in a sample of $Z \rightarrow \mu^{+} \mu^{-}$decays before (black) and after (red) the application of the $Z$ alignment [5].

Tracking efficiencies are estimated using a tag and probe method where the tag is fully reconstructed while the probe is reconstructed excluding hits in the subdetector whose performance are being tested [6]. The method is applied on a sample of $J / \psi \rightarrow \mu^{+} \mu^{-}$decays because of the high statistics available and the capability of the muons of traversing the whole detector, leaving a distinctive signature. The sample is selected using dedicated trigger lines from the TurboCalib calibration stream. Figure 3 shows the muonic tracking efficiencies for data and simulation relative to the year 2018. The dependencies on the track momentum $(p)$ and pseudorapidity $(\eta)$, the number of primary vertices $\left(\mathrm{N}_{\mathrm{PV}}\right)$ and the number of hits in Scintillating Pad Detector $\left(\mathrm{N}_{\mathrm{SPD}}\right.$ hits $)$ are investigated. The efficiencies are above $95 \%$, with a $99 \%$ agreement between data and simulation. Similar performance is observed across the years [7]. A dedicated strategy has been developed to measure the electron reconstruction efficiencies [8]. Electrons behave differently than muons because of the large amount of energy loss in the material due to Bremsstrahlung, which mainly affects the reconstruction performance after the magnet. The strategy consists in a tag and probe method using $B^{+} \rightarrow J / \psi\left(e^{+} e^{-}\right) K^{+}$decays, where the tag is represented by the electron-kaon pair, and the spare electron is the probe.

\section{Particle identification performance}

The information available for the PID is different for charged and neutral particles. For charged PID the information from all the subdetectors is grouped into a single powerful observable. The 

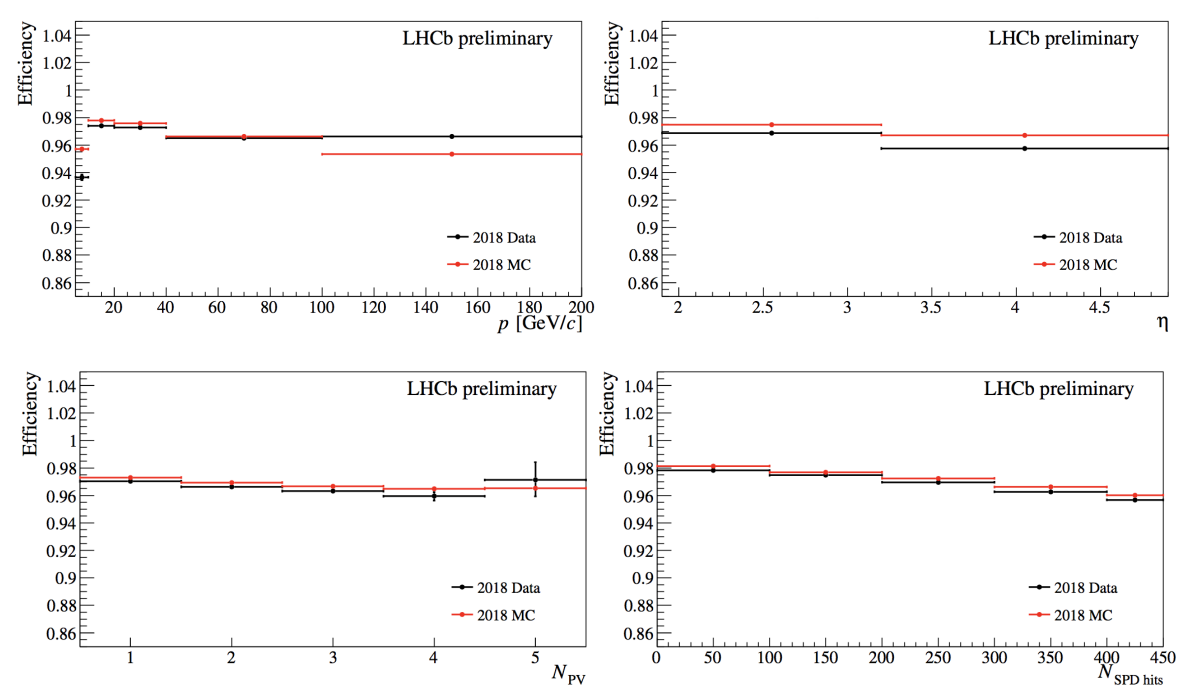

Figure 3: Track reconstruction efficiency versus momentum $(p)$, pseudorapidity $(\eta)$, number of hits in Scintillating Pad Detector ( $\left.\mathrm{N}_{\text {SPD hits }}\right)$ and number of primary vertices $\left(\mathrm{N}_{\mathrm{PV}}\right)$ for 2018 data, and for simulation [7].

$\triangle \mathrm{LL}$ is defined as the difference between the log likelihood of a certain PID hypothesis relative to the hypothesis of that track being a pion. The second variable, ProbNN, is obtained by training multivariate classifiers with simulation including information from the PID subdetectors and the tracking system, and can be interpreted as the probability for a specific PID hypothesis. Because the PID response is not perfectly described in the simulation, using Monte Carlo events to calculate the PID efficiencies would introduce large systematic uncertainties. Instead, data-driven methods are used on calibration samples. Similarly to what happens with the tracking performance, the PID calibration samples are selected using dedicated trigger HLT2 lines in the TurboCalib stream [9]. They are characterised by high purity and statistics, low-multiplicity final state, and large branching fractions. For the figures in this document, samples of pion and kaon tracks are taken from the $D^{0} \rightarrow K^{-} \pi^{+}$candidates originating from decays of $D^{*+}$. Proton tracks are taken from decays $\Lambda_{c}^{+} \rightarrow p K^{-} \pi^{+}$. Figure 4 shows the kaon (top) and proton (bottom) identification efficiencies (in red) and pion misidentification rates (in black) in 2018 data, over a wide momentum range, for two different cuts on the $\Delta \mathrm{LL}$ and ProbNN variables. The looser cut ensures higher signal efficiency while the tigher cuts is characterised by higher background rejection. Figure 5 shows the kaon identification efficiency and pion mis-ID rate as a function of track momentum for tight cuts on both $\triangle \mathrm{LL}$ and ProbNN variables, compared in different years of Run 2. Changes in the tracking performance since 2017 can explain the observed differences [10].

For neutral PID dedicated neural networks can be trained on simulation to separate photons from electrons, hadrons and neutral pions. The corresponding output variables are called isNotE, isNotH, isPhoton, respectively. Figure 6 shows the response of the isNotE (left) and isNotH (right) classifiers. There is work in progress to provide data-driven efficiencies for neutral PID. In 2017, the same production strategy used to select the charged PID calibration samples was extended to neutral PID samples, increasing the phase space coverage with respect to Run 1. 

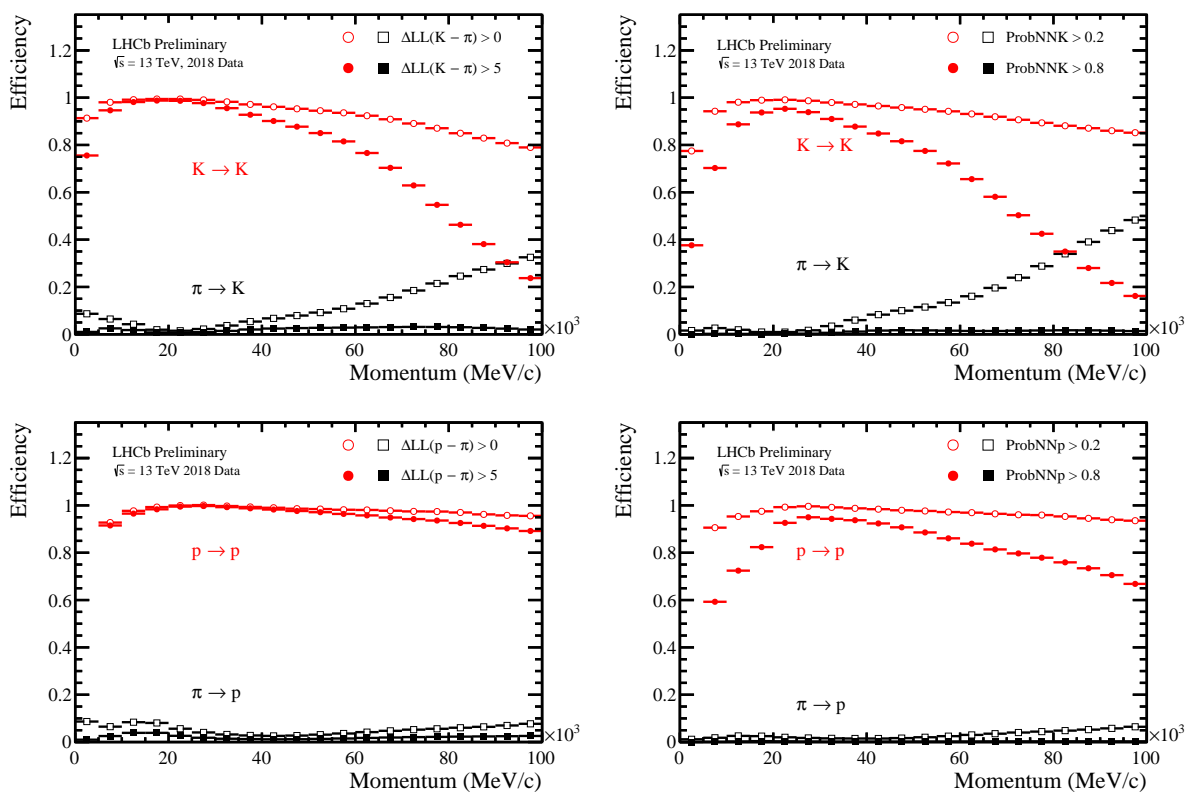

Figure 4: Performance of the loose and tight cuts on the $\Delta \mathrm{LL}$ (left) and ProbNN (right) variables, as a function of track momentum, for (top) kaon-pion separation, (bottom) proton-pion separation in $2018 \mathrm{LHCb}$ data. Identification efficiency is shown in red, while misidentification rate is shown in black for the year $2018[10]$.
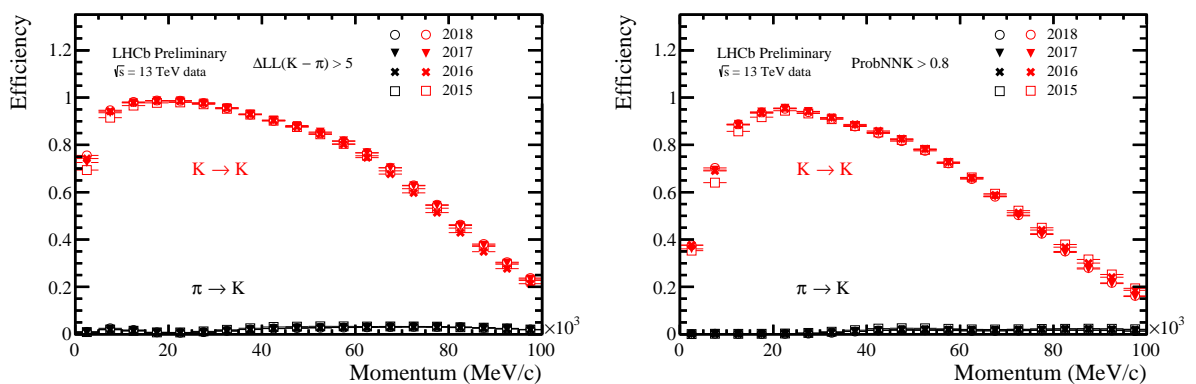

Figure 5: Kaon identification efficiency (red) and pion misidentification rate (black) in 2015-2018 [10].
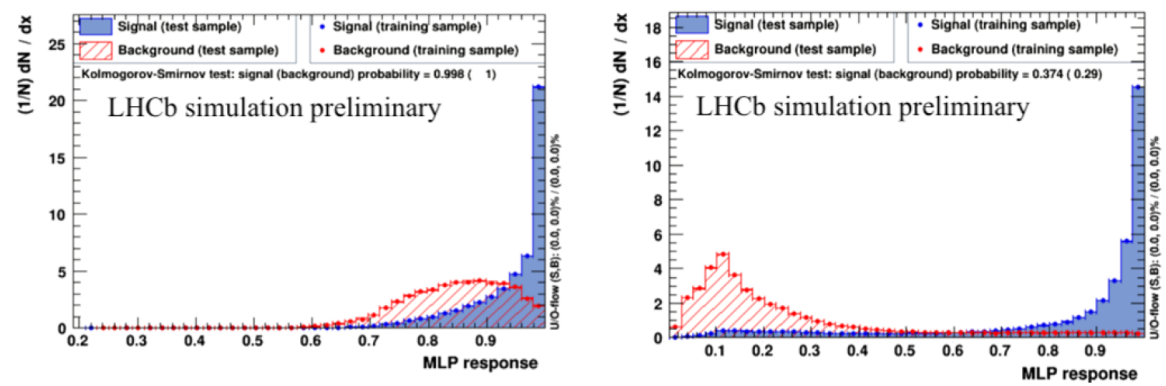

Figure 6: Response of the isNotE (left) and isNotH (right) classifiers with reconstructed photon candidates matching the generated photons (blue) and reconstructed photons matching generated electrons, and neutral pions (red), respectively [11]. 


\section{Conclusions}

Precise alignment, calibration and particle identification performance are crucial for the $\mathrm{LHCb}$ physics programme. $\mathrm{LHCb}$ is the first experiment to adopt a real time detector alignment and calibration with full event reconstruction available online, with online reconstruction quality matching that of the offline reconstruction. This allows the selection of high purity and high statistics calibration samples for the study of PID and tracking performance. The muon reconstruction efficiencies, determined using a tag and probe method, are well above 95\%. A new strategy to determine electron reconstruction efficiencies has also been developed. Excellent PID performance is achieved over the full Run 2. Data-driven calibration methods are introduced to determine the PID efficiencies, reducing the systematic uncertainties due to efficiency mismodelling in the simulation. The PID performance for different charged particles species shows a high discriminating power over a wide momentum range. Neutral PID performance via the use of multivariate classifiers is also described. The excellent results achieved in Run 2 lay the foundation for the challenges that $\mathrm{LHCb}$ will face in Run 3.

\section{References}

[1] LHCb Collaboration, The LHCb Detector at the LHC, JINST 3, 8005 (2008).

[2] LHCb Collaboration, Performance of the LHCb trigger and full real-time reconstruction in Run 2 of the LHC, JINST 14 (2019) P04013.

[3] S. Borghi, Novel real-time alignment and calibration of the LHCb detector and its performance, Nucl. Instrum. Meth. A845 (2017) 560.

[4] W. Hulsbergen, The global covariance matrix of tracks fitted with a Kalman filter and an application in detector alignment, Nucl. Instrum. Meth. A600 (2009) 471.

[5] LHCb Collaboration, Impact of the $Z \rightarrow \mu^{+} \mu^{-}$alignment on the $Z$ mass resolution at $L H C b$, LHCB-FIGURE-2020-009.

[6] LHCb Collaboration, Measurement of the track reconstruction efficiency at LHCb, JINST 10 (2015) P02007.

[7] LHCb Collaboration, Muon track reconstruction efficiencies at LHCb, LHCB-FIGURE-2020010 .

[8] LHCb Collaboration, Measurement of the electron reconstruction efficiency at LHCb, JINST 14 (2019) P11023.

[9] LHCb Collaboration, Selection and processing of calibration samples to measure the particle identification performance of the LHCb experiment in Run 2, EPJ Techn Instrum 6, 1 (2019).

[10] LHCb Collaboration, Global PID performance for charged particles in Run 2 of LHCb, LHCB-FIGURE-2020-012.

[11] LHCb Collaboration, Reconstruction, calibration, and PID performance for photons and neutral pions, LHCB-FIGURE-2020-011, in preparation. 